\title{
Adoption and Diffusion Rule of LBS in the View of Social Network Privacy-A Case Study of SinaMicroblogLBS
}

\author{
Xia Chen, Tingjie Lu, Longfei Guo, Yu Shen and Chenguang Wang \\ School of Economics and Management, Beijing University of Posts and \\ Telecommunications, Beijing 100876, China \\ cxbupt@263.net,lutingjie@263.net,guolongf@bupt.edu.cn, zly-951@163.com, \\ 694033972@qq.com
}

\begin{abstract}
Some studies have shown that the attitude and intention of using mobile LBS istrongly influenced by the privacy factor. Since LBS bears prominent privacy attribate, our study selects the aggregation form based on Microblog content in the LBS of SinaMicroblog, namely, LBS Microblog, asthe object of study, acquires an actual Microblog user data sample of over 30,000 items, with over 8.64 million Mioroblog pastings and 20 million Microblog re-postings of these users, conducts a contrastive research on the adoption behavior in LBS of users at Hub nodes and common nodes thecial network through relative analysis, and discusses the diffusion rule of LBS through BASS model. The study finds that the structure attribute of users in social network has strong influence on the adoption rule of LBS; the adoption of different types of $\triangle B S$ by users is obviously in consistency; LBS is remarkably influenced by emergencles related to privacy.
\end{abstract}

Key words: Social network, Mi roblog, LBS, adoption, diffusion rule

\section{Introduction}

LBS is short for Location Based Service, which is a kind of value added service [1] on the basis and with the support of GIS (Geographic Information System), acquiring position information of mobile term nal users through telecommunication network or external location mode and providing services for users. Till December 31, 2012, registered users of SinaMicroblog has exceeded 503 million, it is an important platform for the study of LBS. In SinaMicroblog, geographical location information is a part of Microblog content, and is saved in parallel with "Microblog content", "Microblog picture", etc., mainly including the following three aggregation forms: (1) aggregation based on Microblog content, namely, common LBS Microblog; (2) Microblog aggregation based on pictures, namely, LBS pictures with geographical information, (3) content aggregation based on geographical information, namely, extended service on the basis of geographical information.

Though the industry generally believes that LBS is promising, there are also some problems of privacy disclosure [2-3]. For the study of LBS in the view of privacy, some scholars expound privacy protection methods from the perspective of technology [2-3]; and some scholars focus on the influence of privacy concern on LBS. For example, Zhang Zhijie, etc., point out that the attitude and intention of using mobile LBS is strongly affected by the privacy factor [4]. Chai Yanwei, etc., present that protection of individual positioning privacy is the key issue limiting LBS study and individual privacy problem caused by mobile phone positioning once led to a period of stagnation in development of LBS[5].

The prominent privacy attribute of LBS results in the distinction with other service in SinaMicroblog in terms of adoption and diffusion rule. The existing studies on LBS 
adoption mainly center on the influence of privacy concern on adoption behavior of users by means of structural equation [6,7]. However, in the existing studies, scholars study the diffusion rule in social network, utilizing BASS model [8] etc., there are much less studies on diffusion rule of LBS.

The study selects the aggregation form based on Microblog content in the LBS of SinaMicroblog, namely, it takes LBS Microblog as its object of study, acquires an actual Microblog user data sample of over 30,000 items, with over 8.64 million Microblog postings and 20 million Microblog re-postings of these users, conducts a contrastive researchon the adoption behavior in LBS of users at Hub nodes and common nodes in social network through relative analysis, and discusses the diffusion rule of LBS through BASS model.

The rest parts of the study is organized as follows: the second part discusses data acquisition; the third part analyzes the adoption behavior in LBS at Hub nodes and common nodes in social network; the fourth part analyzes LBS network structure features and studies the diffusion rule of LBS in the view of privacy by using BASS model.

\section{Data Acquisition}

The data set used in this study all comes from SinaMicroblog (htp://weibo.com/). Microblog crawlers are written in Java language, and then the data is acquired from the API interface authorized by Sina.

In Microblog, the relationship between users can be follower, following as well as mutual following, among which follower and following are unidirectional and belong to weak ties proposed by Granovetter, while the relationship of matual following can be kept up only by mutual acceptance of each other and belongs to strong tie. In this study, in consideration of specific research questions, only the bidirectional friend relationship (mutual following) is acquired.

The acquiring process starts from a randon user, and breadth-first traversal algorithm is applied to acquire friends who build mutual friend relationship with the user; the process is repeated again and again, so as to acquire four layers of user data with this relationship (namely, the starting point A. friends of A, friends of A's friends, and friends of A's friends' friends). A total data sample of over 30,000 items has been acquired, with over 8.64 million Mieroblog postings and 20 million Microblogre-postings, with the whole process lasting for four months.

\section{Analysis on the Adoption Behavior of LBS}

\subsection{LBS Adoption Behavior of Hub Nodes}

It is generally believed that there are a few nodes with particularly large linking number in an online complex network; these nodes often provide information for others in interpersonal communication network, are "activists" exerting influence on others, play an impotant role as intermediary agent or filtration in the process of mass communication effect, and diffuse information to audiences, thus forming the two-step flow of conmunication of information transfer. These nodes are called Hub nodes.

Here, a crucial conclusion is proposed first: Hub nodes are more likely to conduct adoption behavior in the early phase-stage of diffusion process, however, it is not because Hub nodes are more innovative orrisk-taking than common nodes but because the service appears in a larger number of times before Hub nodes' eyes [9]. In addition, stars pay special attention to their privacy, especially their locations; in a similar way, Hub nodes or star nodes concern more about their privacy. In regard to LBS Microblog with privacy attribute, some innovation nodes conduct adoption of the service in the early phase since LBS has emerged. However, when Hub nodes have noticed the emergence of new LBS business, they won't take the lead to make adoption; when a great deal of friends of hub 
nodes have conducted adoption, the privacy disclosure of LBS also shows up. Hence, the study hypothesizes that:

$\mathrm{H}_{1}$ : Hub nodes are less likely to adopt LBS than common nodes.

In order to verify the hypothesis, first it is necessary to define Hub nodes. According to the requirements of normal distribution on confidence interval, the study defines Hub nodes as nodes with number of degrees twiceas much as the standard deviation above the expectation. In order to prevent the fluctuation of the result caused by the inaccuracy of definition, the study takes the nodes whose amount of friends are among the top $2.5 \%$ for reference. Moreover, the studyconsidersusers' first posting of LBS Microblog as their adoption of the LBS of SinaMicroblog. The result is shown in Table 1.

Table 1. Contrast of LBS Adoption Rate of Various Types of Users

\begin{tabular}{|c|c|c|}
\hline Type & User Sample Number & Adoption Rate \\
\hline Common Nodes & 32312 & $54.87 \%$ \\
\hline $\begin{array}{c}\text { HubNodes(twice as much as } \\
\text { the standard deviation) }\end{array}$ & 1616 & $34.5 \%$ \\
\hline HubNodes(top 2.5\%) & 808 & $29.8 \%$ \\
\hline
\end{tabular}

Table 1 indicates that the LBS adoption rate of Hub nodes $(345 \%, 29.8 \%)$ is far less than that of common nodes (54.8\%); along with the increase of the number of degrees of users, the adoption rate is in a downward trend. Thus, the hypothesis is verified:

Due to the influence of privacy attribute, Hub nodes are less likely to adopt LBS than common nodes.

\subsection{LBS Adoption Behavior of Common Nodes}

Common SinaMicrobloguser are the uppermost group in Microblogs, and the important target of Microblogmarketing of every enterprise and merchant. The study on behavior trend of common nodes is helpfulfor Microblog enterprises to enhance the activeness of users and conduct differentiated marketing on users specifically. GuoLongfei points out that the adoption behavior of a certain service is influenced by the number of friends adopting this service[10]. Although LBS has the prominent privacy attribute, the level of sensitivity on privacy disclosure of most common nodes is obviously lower than that of Hub nodes at the present phase. Therefore, the studyhypothesizes that:

H2: The adoption of LBS of common nodes is influenced by the number of its friends adopting the business.

Correlation analysis is applied to verify this hypothesis. Since the number of friends and other data are unordered and there is no linearly dependent relation between the two, the study selects Spearman correlation coefficient to verify the correlation of the two. The general computational formula of Spearman correlation coefficient is:

$$
r_{S}=1-\frac{6 \sum_{j=1}^{n}\left(R_{j}-Q_{j}\right)^{2}}{n\left(n^{2}-1\right)}
$$

Among it, $R_{j}$ and $Q_{j}$ respectively are the ranks in No.j values of the two variables. The closer $\left|r_{S}\right|$ is to 1 , the higher the degree of correlation is; the closer $\left|r_{S}\right|$ is to 0 , the lower the degree of correlation between the two variables is. If $r_{s}>0$, it shows that there is a positive correlation between the two variables; if $r_{s}<0$, it shows that there is a negative correlation between the two variables. In line with the value of $\left|r_{s}\right|$, the degree of correlation can be divided into four grades:

1. When $0.8 \leq\left|r_{s}\right|$, there is a high correlation between the two variable; 
2. When $0.5 \leq\left|r_{S}\right| \ll 0.8$, there is a moderate correlation between the two variables;

3. When $0.3 \leq\left|r_{s}\right| \ll 0.5$, there is a low correlation between the two variables;

4. When $\left|r_{s}\right| \ll 0.3$, there is a weak correlation between the two variables;

The significance level reflects the possibility of making mistakes while rejecting a certain original hypothesis. The study sets the significance level as 0.01 for the verification; namely, on the occasion that the original hypothesis is right, the possibility for the researcher to accept the hypothesis is $99 \%$. The result of Spearman correlation analysis is as follows:

\section{Table 2. Correlation Coefficient of the Number of LBS MicroblogPostings and the Number of Friends Adopting LBS Business}

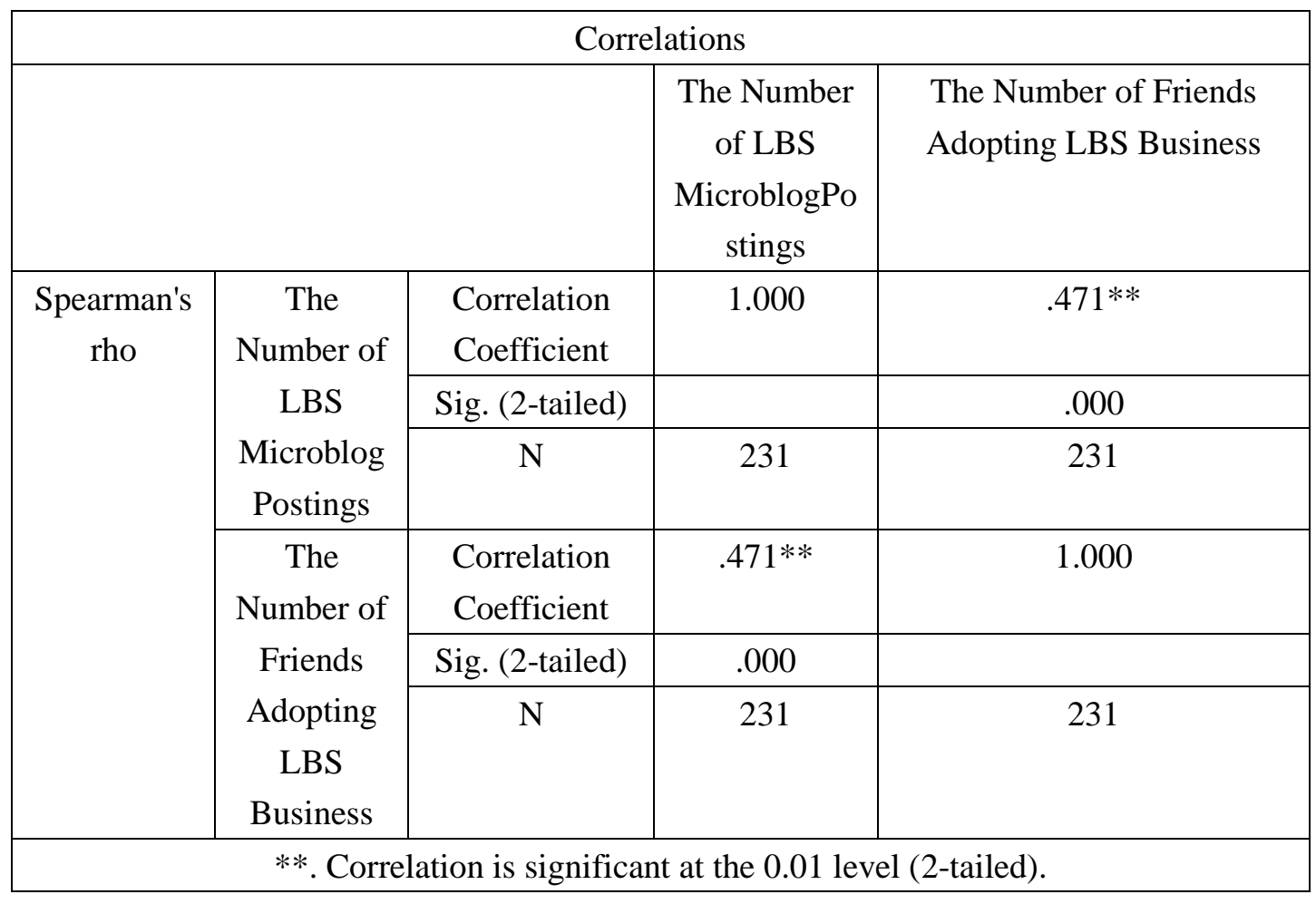

Table 2 shows that there is a moderate positive correlation of 0.471 between the number of LBS Miroblog postings and the number of friends adopting LBS business; that is, the number of friends adopting LBS influences the number of LBS Microblog postings. On the other hand, the number of LBS Microblog postings also influences the number of friends adopting LBS business.

It can be seen from this point that for common user nodes, there is certain influence of the privacy attribute of LBS on users, but the influence is not evident. The study tries to analyze the influence of the privacy attribute on common nodes from other aspects. As Guolongfei indicates [10], the number of Microblog postings by users of SinaMicroblog satisfies the power-law distribution of $r=2.74$; since the influence of LBS privacy attribute is not evident, the number of LBS Microblog postings of common nodes shall also satisfy similar rule. The study, through research and statistics, finds out that the number of LBS Microblog postings of common nodes also satisfies the power-law distribution of $\mathrm{P}(\mathrm{k})$ $\sim \mathrm{K}^{-\mathrm{r}}$, and $\mathrm{r}=1.10$ is obtained through linear fitting, as shown in Graph 1: 


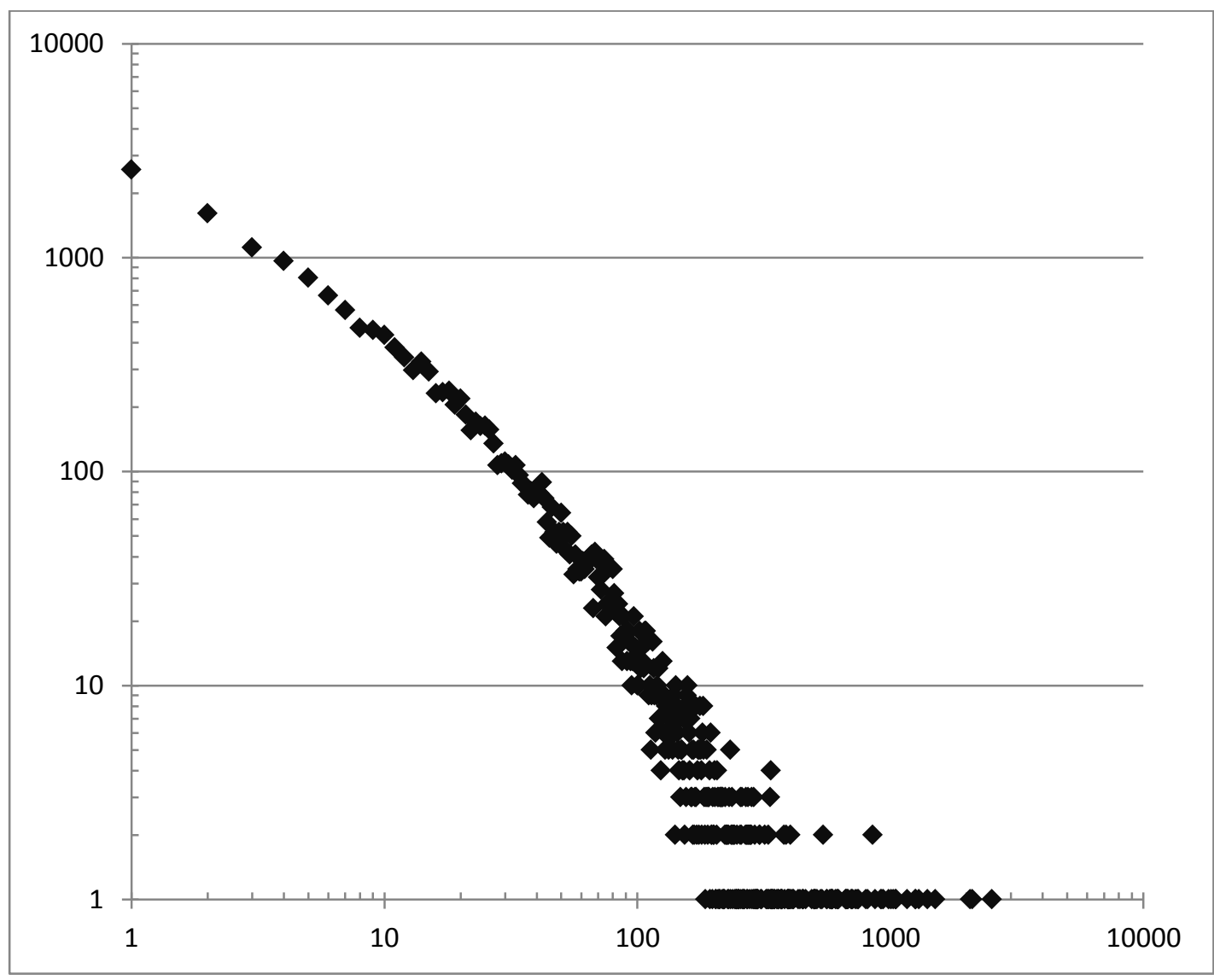

\section{Graph 1. Distribution Graph of the Number of LBS MicroblogPostings of Common Nodes}

LBS of SinaMicroblogconsist of threetypes: aggregation based on Microblog content, Microblog aggregation based on pictures and content aggregation based on geographical information. Since the three types of service all belong to LBS and possess strong privacy attribute, the study holds that there is strong correlation between the three types of business; that is on even ground, users who often use one of three LBS businesses are very likely to use other BBS businesses. For the convenience of research, the study chooses the former tog types of businesses to discuss.

$\mathrm{H}_{3}$ : Common users possess behavior consistency on LBS relevant business.

In order to Verify this hypothesis, the patterns of manifestation of the two types of businesses reed to be found first. Aggregation based on Microblog content is the most common LBS Microblog; in accordance with the above statement, the adoption degree of it can-be presented by the number of LBS Microblog postings of users. Microblog ggregation based on pictures shall be presented by the number of LBS pictures posted by users. Hence, the problem is converted to the study on the relation of the number of LBS Microblog postings and the number of LBS pictures of users. The analysis result utilizing Spearman correlation coefficient is as follows: 
Table 3. Correlation Coefficient of the Number of LBS MicroblogPostings and LBS Pictures

\begin{tabular}{|c|c|c|c|c|}
\hline \multicolumn{5}{|c|}{ Correlations } \\
\hline & & & $\begin{array}{l}\text { The Number of } \\
\text { LBS Pictures }\end{array}$ & $\begin{array}{l}\text { The Number of LBS } \\
\text { MicroblogPostings }\end{array}$ \\
\hline \multirow[t]{6}{*}{$\begin{array}{l}\text { Spearman } \\
\text { 's rho }\end{array}$} & \multirow[t]{3}{*}{$\begin{array}{l}\text { The Number of } \\
\text { LBS Pictures }\end{array}$} & $\begin{array}{l}\text { Correlati } \\
\text { on } \\
\text { Coefficie } \\
\text { nt }\end{array}$ & 1.000 & $.902 * *$ \\
\hline & & $\begin{array}{c}\text { Sig. } \\
\text { (2-tailed) }\end{array}$ & & 0.000 \\
\hline & & $\mathrm{N}$ & 32312 & 32312 \\
\hline & \multirow[t]{3}{*}{$\begin{array}{l}\text { The Number of } \\
\text { LBS } \\
\text { MicroblogPosti } \\
\text { ngs }\end{array}$} & $\begin{array}{l}\text { Correlati } \\
\text { on } \\
\text { Coefficie } \\
\text { nt }\end{array}$ & $.902 * *$ & \\
\hline & & $\begin{array}{c}\text { Sig. } \\
\text { (2-tailed) } \\
\end{array}$ & & \\
\hline & & $\mathrm{N}$ & & 32312 \\
\hline
\end{tabular}

The above table indicates that there is a high positive correlation of 0.902 between the number of LBS pictures and the number of LBS Microblog postings; that is, users who often post LBS Microblog also often pos LBS pictures. This means that when a user who often uses a type of LBS I likely to use another type of LBS business.

In addition, through the research on the number of sign-in sites of users, the study also finds that there is a moderate prsitive correlation of 0.67 between the number of sign-in sites of users and the number of $\mathrm{BS}$ Microblog postings, and there is a moderate positive correlation of 0.634 between the number of sign-in sites of users and the number of LBS pictures. This manifest that a user who likes to post LBS Microblog is willing to sign in at different places; namely, common users possess behavior consistency on LBS relevant business.

\section{Analysis on LBS Diffusion Rule}

\subsection{Structare Characteristics of LBS Network}

Obviously, all LBS users in SinaMicrobloghave constituted a new network. Also, as adopting LBS is more like a common interest, the studyhypothesizes that:

$\mathrm{H}_{4}$ : Useradopting LBS constitutes a small world network with close ties between individuals

Since the characteristics of small world of network are manifested as larger clustering coefficient and smaller average length of the shortest path. To test and verify the hypothesis, only the clustering coefficient of LBS network and average length of the shortest path shall be calculated. Extracting the LBS user set from source data set to form a new network, we, through Pajek analysis, can get that the clustering coefficient of new LBS network is 0.51 and the average length of the shortest path is 5.41; the clustering coefficient of the original network is 0.52 , and the average length of the shortest path is 
4.67; the clustering coefficient of random network is 0.0004 , and the average length of the shortest path is 3.88. Therefore, LBS user group has a closer individual connection, and constitutes a LBS network with the small world characteristics. The various parameters are shown in the following table.

Table 4. Comparison on Parameters of LBS Network and that of Other Networks

\begin{tabular}{|c|c|c|}
\hline Online Social Network & Clustering Coefficient & $\begin{array}{c}\text { Average Length of the } \\
\text { Shortest Path }\end{array}$ \\
\hline LBS Network & 0.51 & 5.41 \\
\hline SinaMicroblog & 0.52 & 4.67 \\
\hline Random Network & 0.0004 & 3.88 \\
\hline YouTube & 0.136 & 5.1 \\
\hline Flicker & 0.313 & 5.67 \\
\hline
\end{tabular}

Through comparison, we can see that, LBS user group is a formed circle on the basis of interest. Namely, LBS users mass in large quantities in the form of circle, and also radiate and diffuse outward with circle as the core. The asers are closely linked and mutually influenced.

\subsection{Diffusion Rule of LBS Business}

LBS diffusion refers to the process that as time goes by, more and more users adopt LBS of SinaMicroblog since its release

There are a great variety of models of innovationdiffusion in terms of marketing and technology forecasting, of which Bass model-is the most famous. Bass(1969) proposed Bass model, and summarizedtwo factors influeneing diffusion speed of new product (new business): One is the innovation factor (external factor), meaning that the ones who have never used the product (business) start to use the product (business) because of mass media and other external factors; the other is imitation factor (internal factor), referring to the interpersonal influence (mainly the oral communication from the adopter of product to the one who does not adopt the product)[21]. Based on the model, the users of LBS are fallen into two groups: One is the buyer only influenced by external factors, called as "innovator"; the other is the buyer only influenced by internal factors, called as "imitator". The formula of Bassmodel is as follows:

$$
\frac{\mathrm{f}(\mathrm{t})}{[1-\mathrm{F}(\mathrm{t})]}=\mathrm{p}+\mathrm{q} * \mathrm{~F}(\mathrm{t})
$$

$f(t)$ refers to the time density function of adopters in the term $t($ the term can be decided according to practical situation), $\mathrm{F}(\mathrm{t})$ refers to the proportion of people who have adopted the ervice to all adopters in the term $t, p$ is innovation coefficient, indicating the possibility of user's adopting the service influenced by innovation coefficient, and q is imitation coefficient, indicating the possibility of user's adopting the service influenced by imitation coefficient. If $\mathrm{m}$ is set as the maximum market capacity of adopters, the number of adopters in the term $t$ can be expressed as:

$$
m * f(t)=n(t)
$$

Then the cumulative adopters are:

$$
\mathrm{m} * \mathrm{~F}(\mathrm{t})=\mathrm{N}(\mathrm{t})
$$


And the cumulative distribution function of S model can be got through the integral of the formula above:

$$
\mathrm{N}(\mathrm{t})=\mathrm{m} *\left[\frac{1-e^{-(p+q) t}}{1+\frac{q}{p} * e^{-(p+q) t}}\right]
$$

Therefore, as long as $\mathrm{N}(\mathrm{t})$ is known, $\mathrm{n}(\mathrm{t})$, the number of non-cumulative adopters can be got through further differential:

$$
\mathrm{n}(\mathrm{t})=\mathrm{m} *\left[\frac{p(p+q)^{2} * e^{-(p+q) * t}}{\left[p+q * e^{-(p+q) t}\right]^{2}}\right]
$$

Through simultaneous equations, we can arrive at:

$$
\mathrm{n}(\mathrm{t})=\mathrm{p} * \mathrm{~m}+(\mathrm{q}-\mathrm{p}) * \mathrm{~N}(\mathrm{t})-\frac{\mathrm{q}}{\mathrm{m}} *[N(t)]^{2}
$$

In the formula above, $\mathrm{n}(\mathrm{t})$ refers to the number of expected adopters in the next cycre of $\mathrm{t}, \mathrm{p}$ is innovation coefficient, $\mathrm{q}$ is imitation coefficient, $\mathrm{m}$ is the largest market capacity, and $\mathrm{N}(\mathrm{t})$ is the number of cumulative adopters at the moment $\mathrm{t}$ among which, $n(t)$ and $\mathrm{N}(\mathrm{t})$ can be got from the historical data of LBS business, while for $\mathrm{p}$, q, and m, parameter fitting[22] are required. The statistics of the cumulative adopters of LBS in history is as follows (only the data of 18 months islisted here):

Table 5. Table of Number of Cumulative Adopters of SinaMicroblog LBS (18 Months)

\begin{tabular}{|c|c|c|c|c|c|c|c|c|c|}
\hline Month & 11.01 & 11.02 & 11.03 & 11.04 & 11.05 & 1.06 & 11.07 & 11.08 & 11.09 \\
\hline Time(t) & 10 & 11 & 12 & 13 & 14 & 15 & 16 & 17 & 18 \\
\hline Cumulant & 247 & 392 & 558 & 726 & 944 & 1225 & 1533 & 1879 & 2252 \\
\hline Increment & 82 & 145 & 166 & 168 & 218 & 281 & 308 & 346 & 373 \\
\hline Month & 11.10 & 11 & 11.12 & 12.01 & 12.02 & 12.03 & 12.04 & 12.05 & 12.06 \\
\hline Time(t) & 19 & 20 & 21 & 22 & 23 & 24 & 25 & 26 & 27 \\
\hline Cumulant & 2598 & 3269 & 4126 & 4913 & 5695 & 6627 & 7413 & 9387 & 73 \\
\hline Increment & 346 & 671 & 857 & 787 & 782 & 932 & 786 & 1974 & 1786 \\
\hline
\end{tabular}

We adopt MATLAB for estimation and curve fitting of parameters, and adopt lsqurvefit function for iterative computation. Fitting curve LBS user number and original LBS user data are shown in Graph 2. 


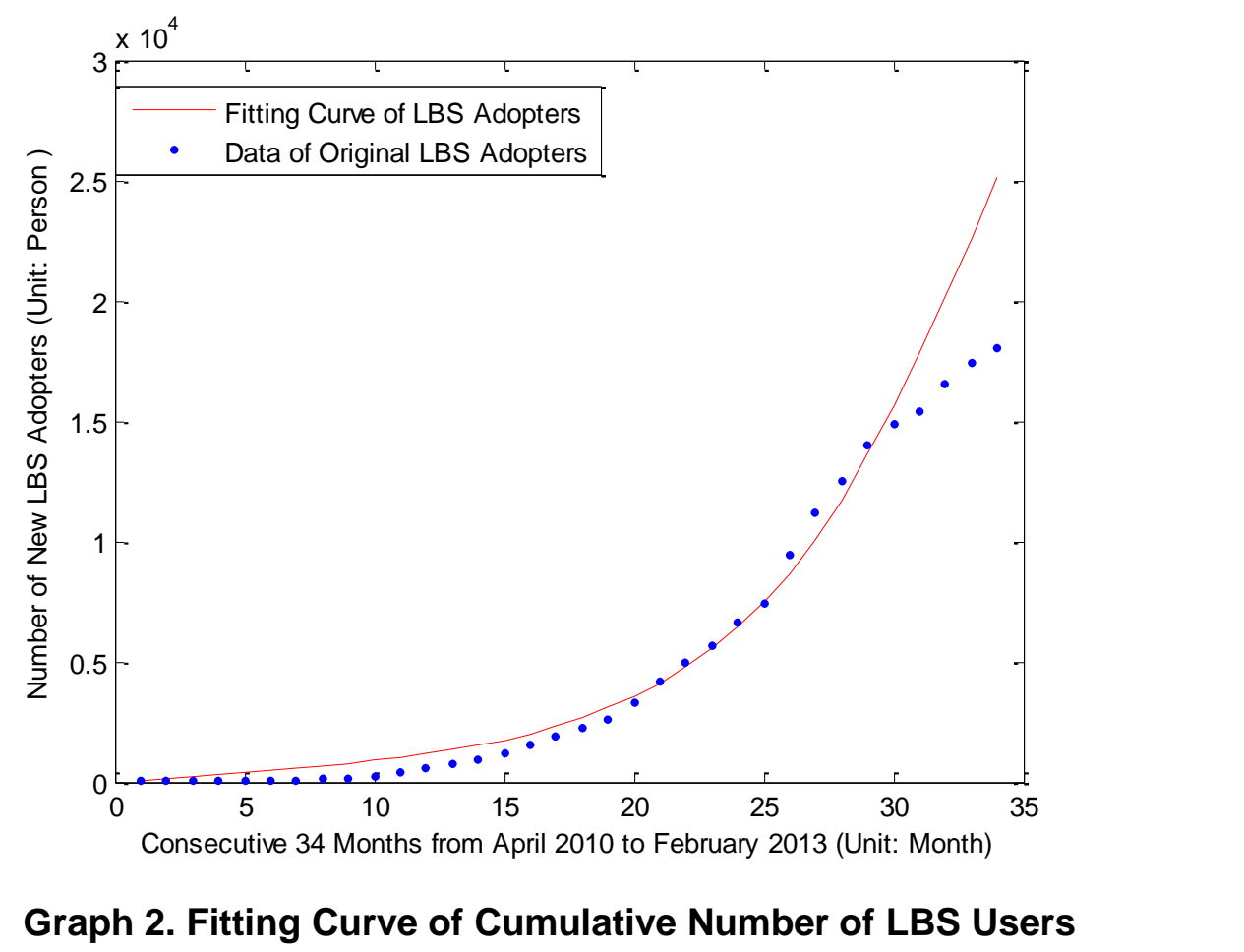

Through MATLAB calculation, the three parameters of $\mathrm{p}, \mathrm{q}$ and $\mathrm{m}$ are as follows:

Table 6. Reference Values

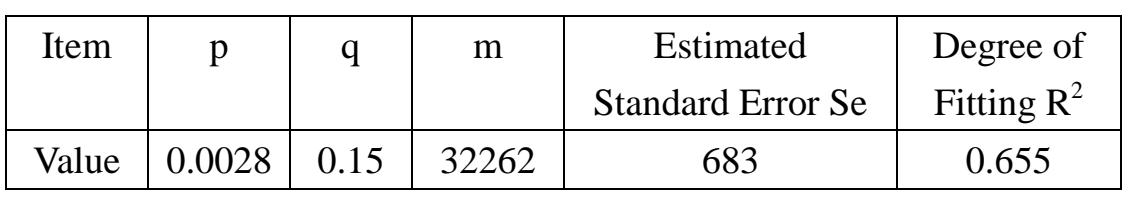

When Yang Jinghui and Wu Chunyou adopt genetic algorithm to conduct parameter estimation for Bass diffusion model, they conclude that, for the growth of Chinese mobile users, the innovation coeffecent is 0.001 , and imitation coefficient is about 0.5 , while for growth of LBS of SinaMicroblog, the innovation coefficient is 0.0028 and imitation coefficient is 0.15 , indicating that the users of internet industry are more innovative than that of communication industry and users are more likely to select the adoption of LBS because of externalfactors.

From Graph 2, we can see that the prediction accuracy is higher in the first 30 months, while there is a separation tendency between the prediction curve and the original data in the next few months. In order to find the reason for separation, we draw the curve of the namber of new LBS users as follows: 


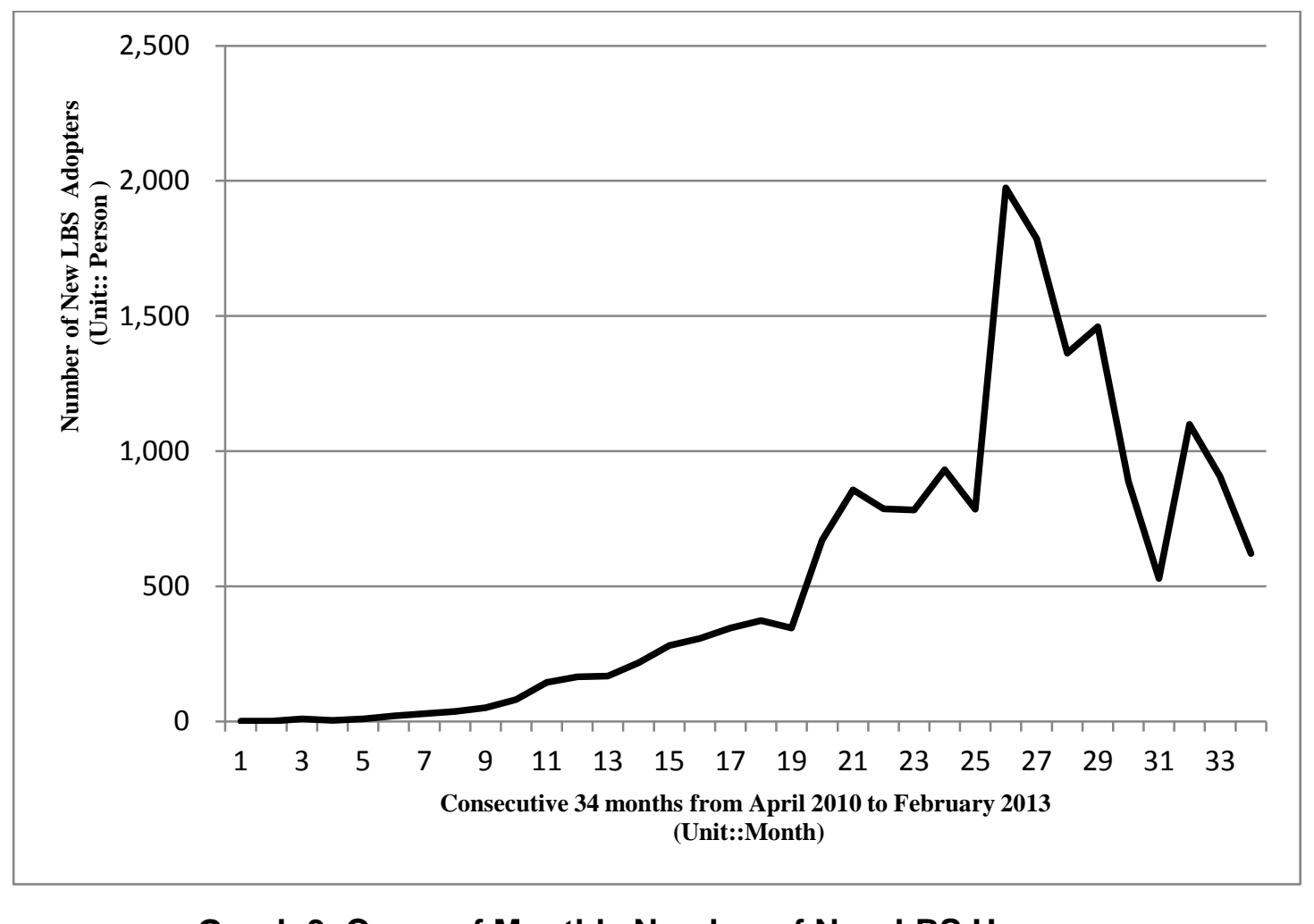

\section{Graph 3. Curve of Monthly Number of New LBS Users}

We can find from Graph 3 that, for the number of new users, there has been fluctuation on a larger scale from May 201270 March 2013 (10 months). In order to verify the fluctuation of the curve, we will draw the number of LBS Microblog postings in the same time period as follows:

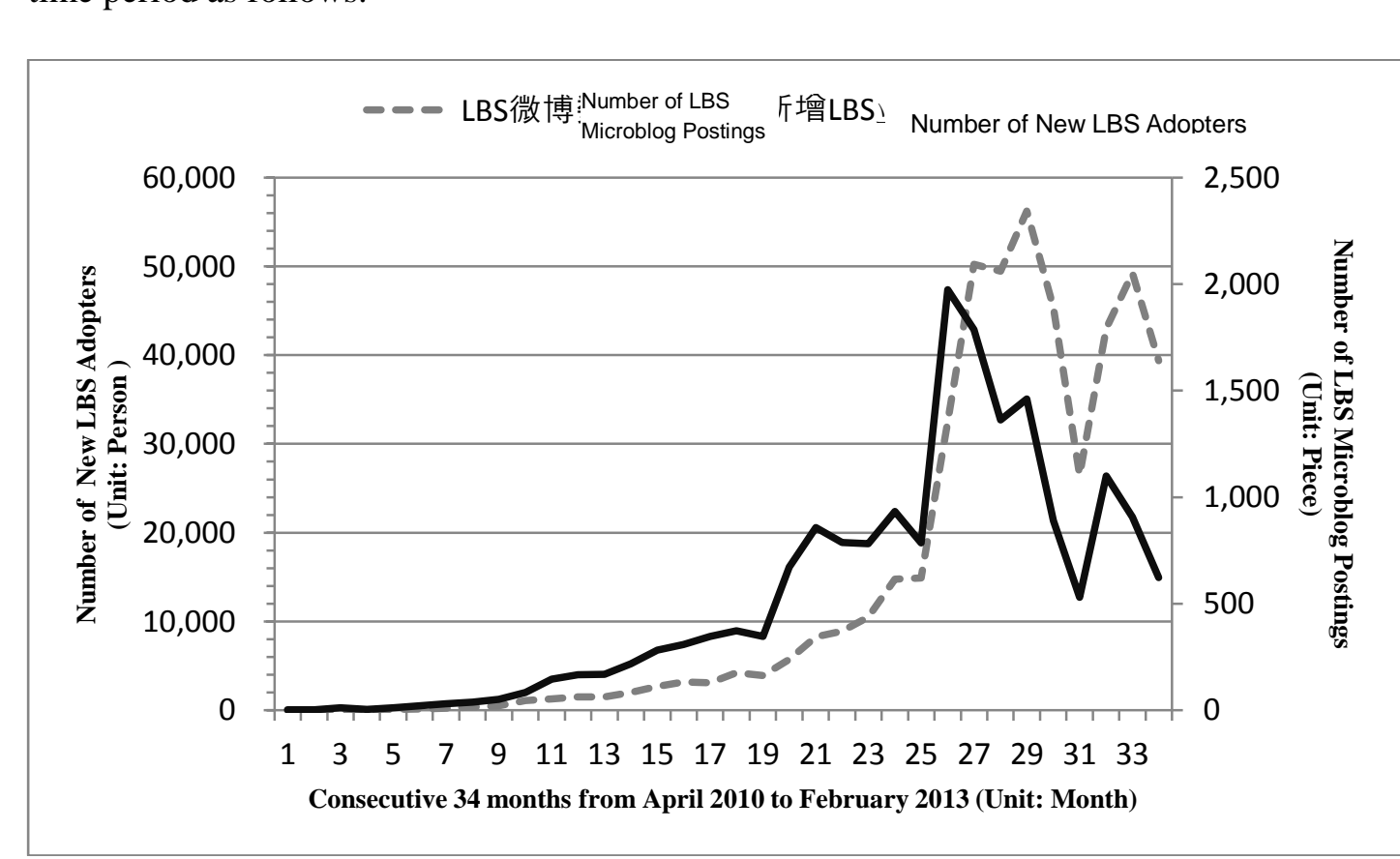

Graph 4. Comparison on the Number of New LBS Adopter and LBS Microblog Postings 
From Graph 4, it is found that there has been the flunctation of similar rules in the same time period for newly increased number of LBS adopters and number of LBS Microblog postings. The two curves present obvious tendency of flunctation, as well as a higher level of similarity. Then we have a preliminary idea that, it might be "emergency" that affects the use of LBS of Sina Microblog users. In order to determine the influence is directed at the user behavior of the whole Sina Microblog or just LBS business, we have analyzed the changes of number of Sina Microblog postings in 2012, as shown below:

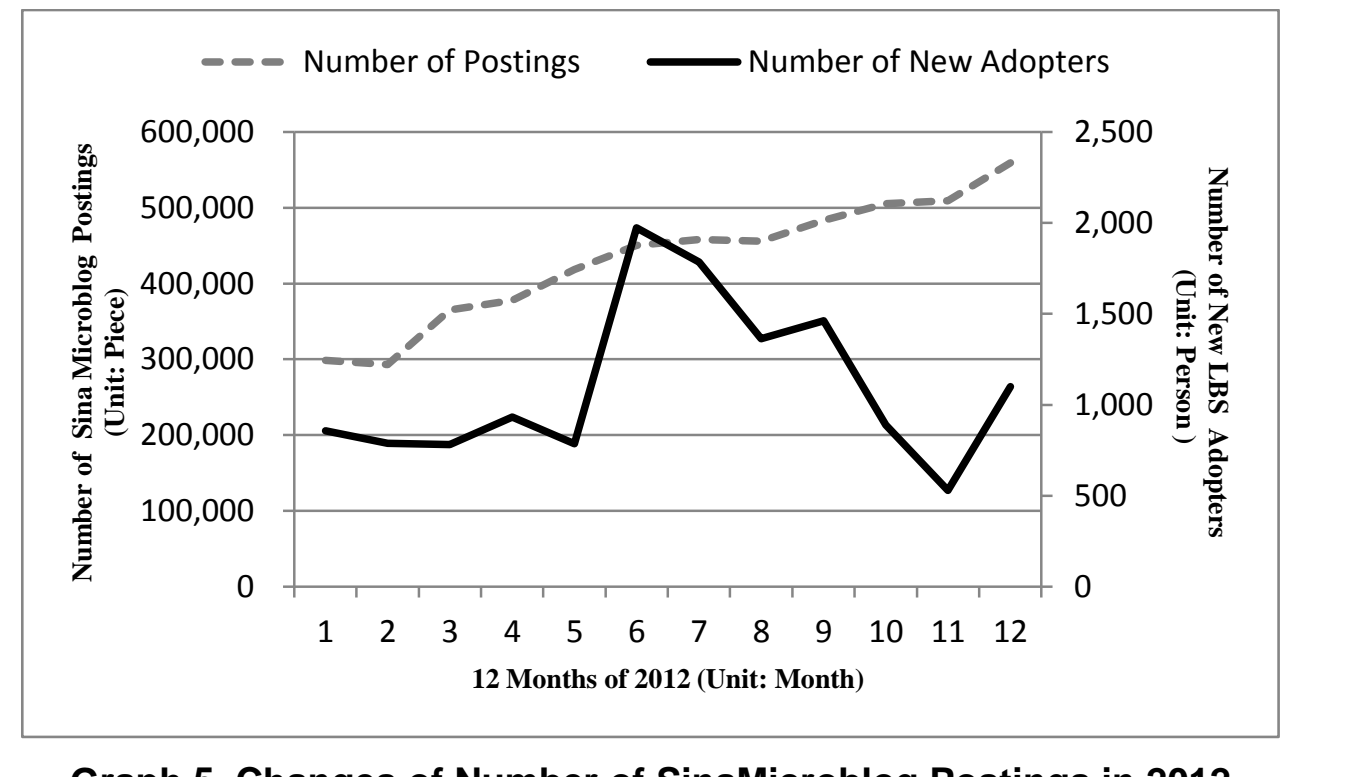

Graph 5. Changes of Number of SinâMicroblog Postings in 2012

It is obviously found from Graph 5 that the number of SinaMicroblogpostings sees an increase and steady trend within the time period, while the number of new LBS users and number of LBS Microblog postings witness significant fluctuation. Therefore, there must be some emergent factors which only affect the development of LBS business, instead of the overall development of the whole Microblog business.

Through the big event statistics of SinaMicroblog since 2012, it shows thatSinaMicroblog opened its LBS platform at the end of April 2012 and the third-party developers could have the access to free LBS service of Sina. Based on its enormous social graph and interest graph of SinaMicroblog, LBS application will get rid of the original "sign-in" pattern, and officially enter into 2.0 times of LBS. This is the greatest event related to the privacy and privacy business within the time period, which is likely to be the main reason for sharp rise of LBS business in May.

However. in November 2012, the express number information of over ten mainstream express enterprises including EMS was disclosed by a large margin, and then the threat of user privacy aroused discussion and attention of broad netizens. Also, Internet Security Forum which was held twice in the same month pushed the discussion on user privacy to a new height. The discussion on privacy was bound to affect SinaMicroblog with complicated relationship and rich content, but the most direct one could be its effect on privacy business, as it might be the direct reason for the sharp drop in Sina LBS in November 2012.

Even though the existing research could not directly relate the privacy behaviors of users of social network and rules of the business to the relevant emergencies of privacy, to some extent, LBS users' attitudes towards LBS are obviously affected by the relevant emergencies of privacy. 


\section{Conclusion}

The study mainly has the following three conclusions: (1)Users' structure attribute in social network has a stronger effect on the adoption rule of LBS business. In the study, it can be found that Hub node is more difficult than common node to adopt LBS business, and users of common node are under the positive influence of their friends in terms of the adoption of LBS business. (2)Common users possess behavior consistency on adoption of various LBS businesses. The conclusion indicates in a practical way that, privacy attribute behavior is basically consistent. The user with a high perception maystay very cautious for a long time, while the user with a low perception may show little attention to privacy threat for long. (3)LBS is remarkably affected by the emergencies of privacy.The business with privacy attribute is remarkably affected by the emergency of privacy, no matter it is the promotion resulted from good news or the reduction led by negative news.

\section{Acknowledgements}

The research is supported by National Basic Research Program of China (973, Program) (2012CB315805), Project of National Natural Science Foundation of China (7172135, 71231002), and the Fundamental Research Funds for the Central/Universities (2013RC0603).

\section{References}

[1] B. Jiangand X. Yao,"Location-based services and GIS in perspective",Computers, Environment and Urban Systems, vol.30,no.6, (2006), pp.712-725

[2] A. Pingley, "A context-aware scheme for privacy-presenving location-based services",Computer Networks,vol.56,no.11,(2012), pp. 2551-2568.

[3] R. Ajami, N. Al Qirim, and N. Ramadan,"Privacy Issues in Mobile Social Networks",Procedia Computer Science,no.10,(2012), pp.672679.

[4] Z. Zhang and T. Lv, "Empirical Study of Users' Aeceptance Model on Mobile LBS", Journal of Beijing University of Posts and Telecommunications(Social Sciences Edition), vol.14, no.1, (2012), pp.56-61.

[5] Y. Chai, Y. Zhao and X Ma, "Mobile Positioning Method for Spatial-temporal behavioral Data Collection and Its Geographical Applications", Areal Research and Development, vol.29, no.6, (2010), pp.1-7.

[6] Z. Tao,"The impact of privacy concern on user adoption of location-based services", Industrial Management \& Data Systems, vol.11, no.2,(2011), pp.212-226.

[7] Z. Tao, An empirical exannimation of user adoption of location-based services", Electronic Commerce Research (2013), pp.1-1

[8] F. Mengand M. He, "Summarization of Research on Bass Model Parameter Estimation Approach," AEROSPACE CONTROL, vol.27, no.1, (2009), pp.104-108.

[9] J. Goldenberg, S. Han and D. Lehmann, et al. "The role of hubs in the adoption processes", Journal of Marketing, vo1.73, no.2, (2009).

[10] L. Guo, "A Study on the Dynamic Influence and Behavior Regularities ofUser' Privacy Concern about Social Network," [D], Beijing University of Posts and Telecommunications, (2013).

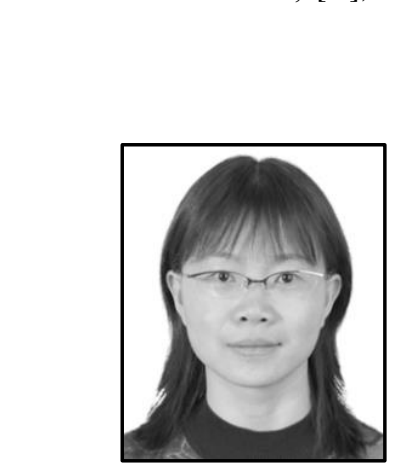

Authors

Xia Chen, she Graduated from Beijing University of Posts and Telecommunications (BUPT), and received a doctor's degree in 2007. She is a Lecturer in School of Economics and Management of BUPT. Her research interests are in the areas of E-Commerce. She has published several papers in the journalsand conferences. TEL: 13901021595, email: cxbupt@263.net 


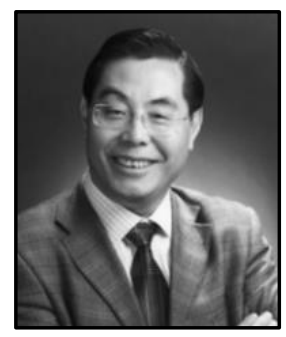

TingjieLv, he is a Professor in Beijing University of Posts and Telecommunications (BUPT). Currently, he is director of the E-Commerce Research Center of BUPT. His current research interests are in the areas of E-Commerceand Communications Management, etc. He has published papers in a number of international journals and conferences.

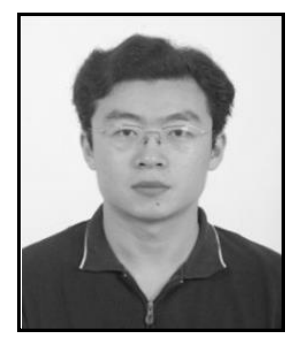

LongfeiGuo, he Graduated from Beijing University of Posts and Telecommunications (BUPT), and received a doctor's degree in 2013. He is a Lecturer in School of Information and Communication Engineering of BUPT.

Yu Shen, he is a graduate student inSchool of Economics and Management of Beijing University of Posts and Telecommunications. His research interests are in the areas of E-Commerce.

Chenguang Wang, He is a graduate sttudent in School of Economics and Management of Beijing University of Posts and Telecommunications. His research interests are in the areas of .social network.

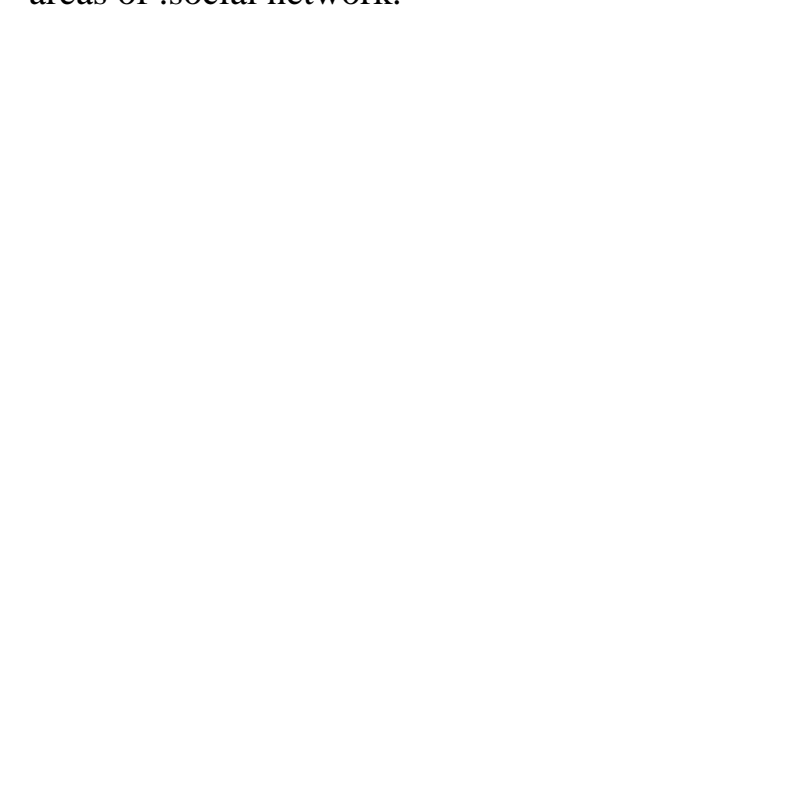


International Journal of Smart Home

Vol. 8, No. 6 (2014)

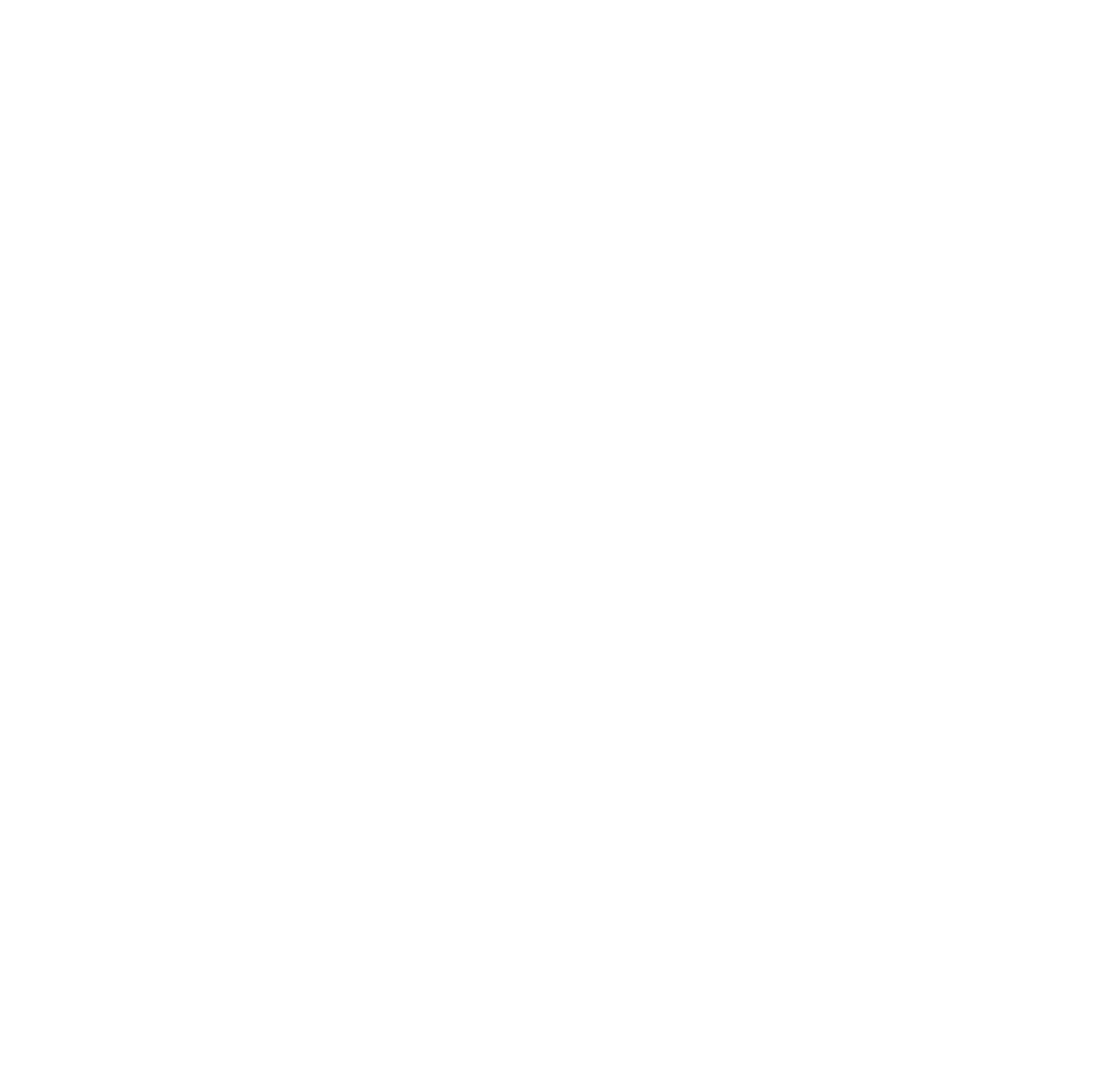

